\title{
IMPLEMENTACIÓN DE UN VISOR GEOESPACIAL EN LA WEB SOBRE RECURSO HÍDRICO PARA EL ACUÍFERO JACÓ, GARABITO, PUNTARENAS, 2014
}

\author{
IMPLEMENTATION OF GEOSPATIAL WEB VIEWER \\ REGARDING THE WATER RESOURCE FOR THE \\ AQUIFER JACO, PUNTARENAS, GARABITO, 2014
}

\author{
Christian Núñez Solis ${ }^{1}$ \\ Universidad Nacional, Heredia, Costa Rica.
}

\begin{abstract}
RESUMEN
Con el propósito de difundir y comunicar con mayor eficiencia investigaciones concernientes al recurso hídrico, se ha implementado una propuesta de Visor Geoespacial en la Web para el acuífero de Jacó de Garabito, Puntarenas, información desarrollada por el Laboratorio de Hidrología Ambiental (LHA) de la Universidad Nacional (UNA) durante los años 2012 y 2014. El sistema se plantea a partir de la importancia del almacenamiento, gestión y comunicación de la información geográfica, como esta debe ser normalizada e interoperable, almacenada y administrada en una base de datos espacial y publicada a través del Internet basado en un Geoportal. Todo esto con el aporte de los programas de código abierto como herramienta para el tratamiento de los datos.
\end{abstract}

Palabras clave: Sistemas de información geográfica, base de datos geoespacial, Geoportal, programas de código abierto, recurso hídrico, Laboratorio de Hidrología Ambiental.

1 Máster en Sistemas de Información Geográfica y Teledetección. Investigador y especialista en SIG del Laboratorio de Hidrología Ambiental. Escuela de Ciencias Biológicas, Universidad Nacional, Heredia, Costa Rica. Correo electrónico: christian.nunez.solis@una.cr/crisnunez80@gmail.com 
Christian Núñez Solis. Implementación de un visor geoespacial en la web sobre recurso hídrico para el acuífero Jacó, Garabito, Puntarenas, 2014

\begin{abstract}
In order to efficiently share and communicate research regarding water resources, a Geospatial Web Viewer application has been implemented with data related to water resources for the aquifer of Jaco, Garabito, Puntarenas. This information was provided by the Laboratory of Hydrology Environmental (LHA, for its name in Spanish) of the Nacional University (UNA) during 2012 and 2014. The system is based on the importance of storing, managing, and communicating geographic information; this is, they way in which this information should be standardized and interoperable, stored and managed in a database space and posted on the Internet based on Web Mapping. All this with the support of open code programs as a tool for processing the data.
\end{abstract}

Keywords: Geographic Information Systems, geospatial database, Geoportal, open source software, water resource, Environmental Hydrology Laboratory.

\title{
Introducción
}

Las tendencias actuales del mapeo se están diversificando siendo cada vez más común que la información geográfica pase del papel a otros medios de difusión como los Geoportales en el Internet. Estas iniciativas son oportunas para que las investigaciones realizadas por entes como las universidades impacten de forma positiva y activa a nivel gubernamental, privado y de población civil, con la posibilidad de ser herramientas útiles para la toma de decisiones y la mejora de la calidad de vida y ambiental de los pobladores.

Ante esta primicia se plantea resolver el problema de la inexistencia de un instrumento adecuado de gestión, administración, consulta, divulgación y visualización de información geoespacial de los proyectos del Laboratorio de Hidrología Ambiental (LHA) que durante 20 años ha crecido como un ente propulsor de la investigación en el tema del recurso hídrico, con productos y resultados de interés público.

El reto es diseñar una propuesta para la gestión del SIG siguiendo el ciclo funcional del dato geográfico, en el que se pueda producir herramientas de información de interés para los usuarios del laboratorio y del público en general, echando mano del aporte que brindan los programas de código abierto y de instrumentos como el Internet para la divulgación de los productos geográficos informativos desarrollados en el proyecto "Vulnerabilidad hidrogeológica para el acuífero Jacó" en Garabito de Puntarenas para el año 2014. 


\section{Área de estudio}

El área de estudio del proyecto (ver mapa 1) está localizada en el distrito de Jacó en el cantón de Garabito dentro de la provincia de Puntarenas. En este espacio de investigación el laboratorio delimita una cuenca hidrográfica de nombre "Jacó", compuesta por tres microcuencas que pertenecen a los ríos Pueblo Nuevo (Mona), Copey y Naranjal, donde se localiza el acuífero Jacó.

La ubicación a partir del sistema de coordenadas CRTM 05, determina que el sitio en investigación se encuentra entre 1061000 - 1070000 $\mathrm{N}$ y 429000 - $437000 \mathrm{E}$ o en coordenadas geográfica entre $9^{\circ} 35^{\prime} 40,66^{\prime \prime}$ 940’34,647” norte y $84^{\circ} 38^{\prime} 48,466^{\prime \prime}$ - 84³4'27,373" oeste.

Mapa 1. Delimitación del área de estudio

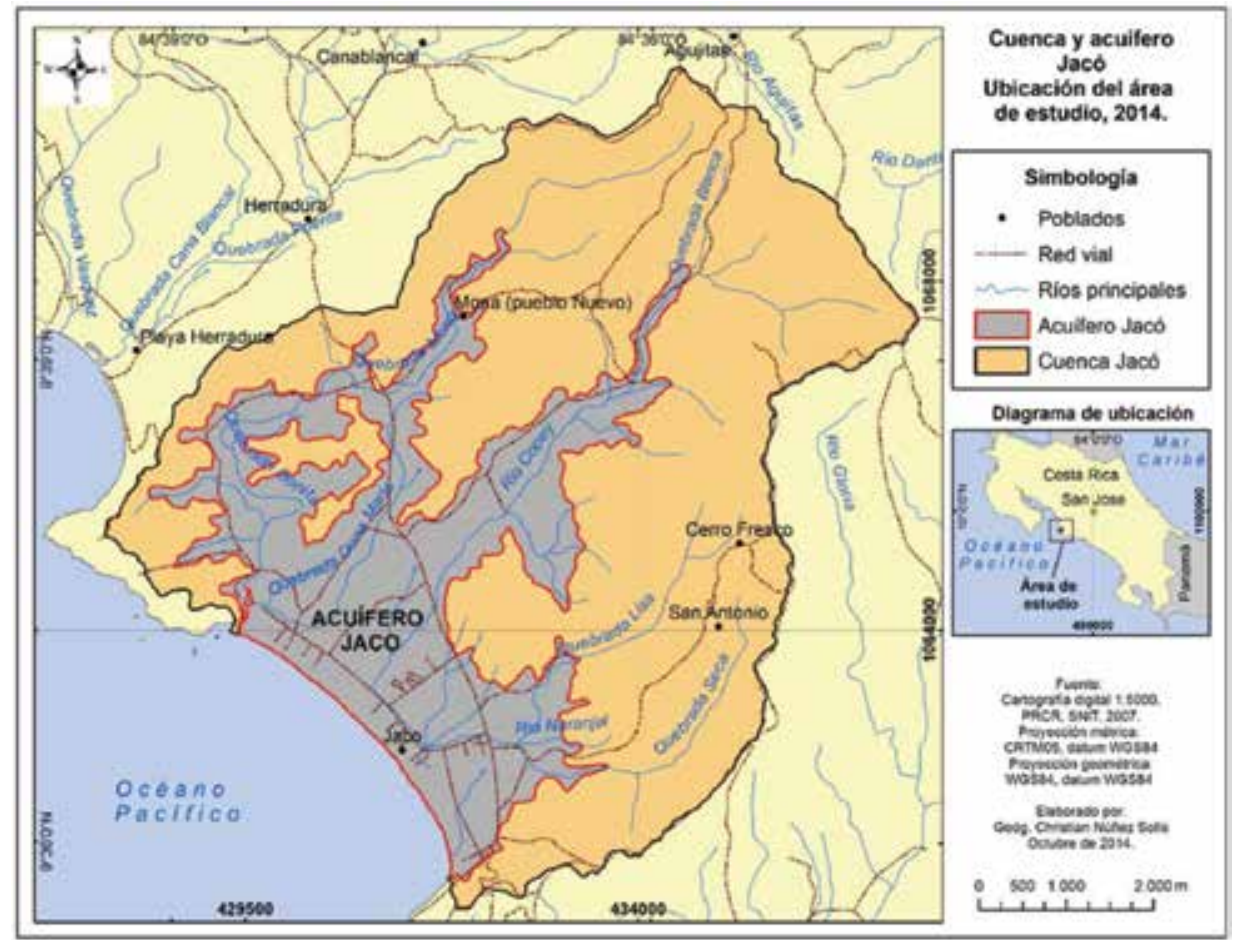

Fuente: Elaboración propia a partir de fuentes externas. 


\section{Marco teórico-conceptual}

El uso del SIG tiene un papel protagónico en las actividades de gestión del recurso hídrico, "el hecho de que aproximadamente un 70\% de la información que manejamos en cualquier tipo de disciplina está georreferenciada" (Olaya, 2011, p.10), presenta a esta herramienta como facilitador del manejo de datos geoespaciales, no solo para mapear y realizar consultas, sino para analizar tendencias y tomar decisiones mediante las aplicaciones que brindan los análisis espaciales (Sánchez y Amoros, 2012). Con la posibilidad de potencializar los datos a través del enlace, base de datos espaciales y publicaciones en páginas web.

Sin embargo, la planificación es la principal metodología para que un SIG logre el éxito deseado. Según Tomlinson (2007), es necesario un estudio previo de organización y gestión de aspectos como: los recursos materiales, apoyos económicos, datos (calidad y cantidad), ejecución y mantenimiento, así como el plan de implementación del escenario idóneo; enmarcado dentro de la gerencia y administración de proyectos en SIG. Algunas de las reglas para diseñar una parte de la planificación se encuentran en los protocolos de gestión de información geográfica, que se desarrollan a partir de la normalización e interoperabilidad, esta acción se encuadra en los estándares de calidad del comité ISO TC-211, cuyo objetivo es "formular un conjunto integral de normas de información geográfica" (Instituto Panamericano de Geografía e Historia -IPGH-, 2010, p.11). Aspectos medulares para que el contenido del visor geoespacial sea eficiente e intuitivo para los interesados.

Tales normas especifican modelos, métodos, herramientas y servicios para la gestión, adquisición, procesamiento, análisis, acceso, presentación y transferencia de datos geográficos digitales entre diferentes usuarios, sistemas y localizaciones. La lista se extiende desde el ISO 19101 al 19134; sin embargo, la siguiente investigación solo toma en cuenta los ISO 19113, 19114 y 19115, los mismos se detallan a continuación:

- $\quad$ Principios de calidad (19113): establece principios para describir la calidad de datos y conceptos geográficos para el manejo de la información de calidad para los datos geográficos (IPGH, 2010, p.57).

- $\quad$ Procedimientos de evaluación de la calidad (19114): proporciona un marco para determinar y evaluar la calidad aplicable a los conjuntos 
de datos geográficos digitales, en forma consistente con los principios de calidad de datos que se definen en la ISO 19113 (IPGH, 2010, p.59).

- $\quad$ Metadatos (19115): define los elementos de metadatos, proporciona un esquema y establece un conjunto común de terminología, definiciones y procedimientos de extensión de los de metadatos (IPGH, 2010, p.61).

Por su parte, otro componente de la planificación recae en el software, para los intereses del siguiente estudio se toma la decisión de trabajar con programas de código abierto, concepto que se define como "el software distribuido y desarrollado libremente" (Open Source.Org, 2014, párr. 1, p.1).

Seguidamente, las bases de datos son componentes esenciales de los sistemas de información geográfica porque en ellas se organiza y almacena el contenido de las aplicaciones que forman parte del sistema. El diseño de las base de datos "es el proceso por el que se determina la organización de una base de datos, considerando su estructura, contenido y las aplicaciones que se han de construir" (Radilla, 2008, p.2). De esta forma, los sistemas gestores de base de datos son la herramienta más adecuada para "almacenar los datos en un sistema de información debido a sus características de seguridad, recuperación ante fallos, gestión centralizada, estandarización del lenguaje de consulta y funcionalidad avanzada". (Olaya, 2011, p.181).

Los SIG se están moviendo de un paradigma aislado, independiente, monolítica y propietario que trabajan en una arquitectura al lado del usuario, hacia aplicaciones y componentes basados en la web más pequeños con una funcionalidad específica de geo-procesamiento y de intercambio transparente de datos entre ellos. La interoperabilidad es el núcleo de este nuevo modelo de servicios web (Anderson y Moreno, 2003, p.1).

Según Olaya (2011), el avance de las redes locales y de Internet "ha permitido que se acceda a la información geográfica contenida en un SIG utilizando el paradigma cliente-servidor. Para ello es necesario contar con componentes en el lado servidor que distribuyan la información y componentes en el lado del cliente para acceder a esta" (p.547). 
Christian Núñez Solis. Implementación de un visor geoespacial en la web sobre recurso hídrico para el acuífero Jacó, Garabito, Puntarenas, 2014

De esta forma, la tecnología de los portales geoespaciales en el Internet, según Gorni, Giannotti, Brito, Knopik y Rodríguez (2007) y Olaya (2011), debe entenderse como un SIG accesible a través de Internet. Esto es lo que se engloba bajo la denominación genérica de Geoportal, Web Mapping, SIG Web o cualquier otro sinónimo. Esta aplicación, es un Sistema de Información (SI) y como tal, generalmente, está compuesto por algunos de los componentes básicos de un SI común, como por ejemplo: lenguaje de desarrollo y un banco de datos (BBDD).

Figura 1. Esquema de un Geoportal

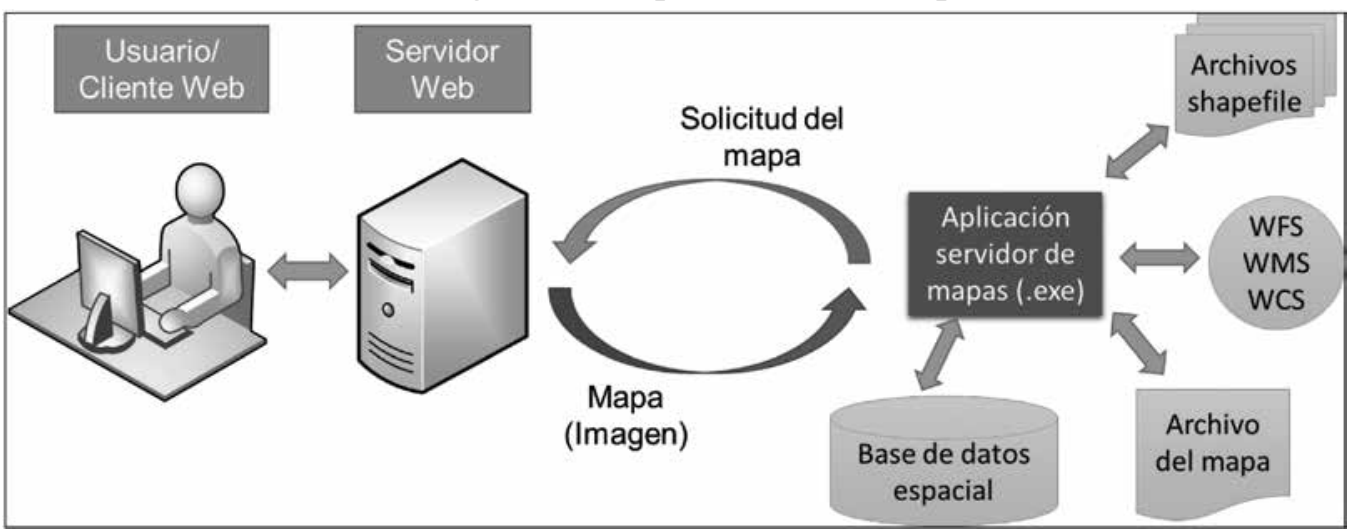

Elaboración propia modificado de Radilla, 2008.

Pero la BBDD de un SIG debe ser capaz de manipular informaciones espaciales. Un buen Geoportal debe tener la capacidad de generar los mapas dinámicamente conforme a las peticiones de los usuarios. Para ello, se hace necesario un servidor de mapas. Así, los componentes, generalmente, encontrados dentro de esta tecnología son: 1) Un Cliente - generalmente un browser de Internet; 2) Un Servidor Web. Ejemplos: Apache, JBoss, IIS, otros; 3) Una Lenguaje de Desarrollo que pueda ser interpretada por el WebServer (HTML, Java, ASP, PHP, otras); 4) Un Banco o Base de Datos Espacial y 5) Un Servidor de Mapas para generar los mapas dinámicamente (ver figura 1).

Algunas de las ideas fundamentales que caracterizan a las tecnologías de Web Mapping y su papel actual son las siguientes: no es necesario un software SIG específico, perfil menos técnico, potencialidad del trabajo corporativo, información actualizada, independencia del sistema, personalización de aplicaciones y combinación de cartografía y otros elementos. 


\section{Marco metodológico}

Dos son las fases esquematizadas para la ejecución de la investigación, presentadas en la figura 2 y 3 , y descritas a continuación:

Figura 2. Esquema general Fase 1

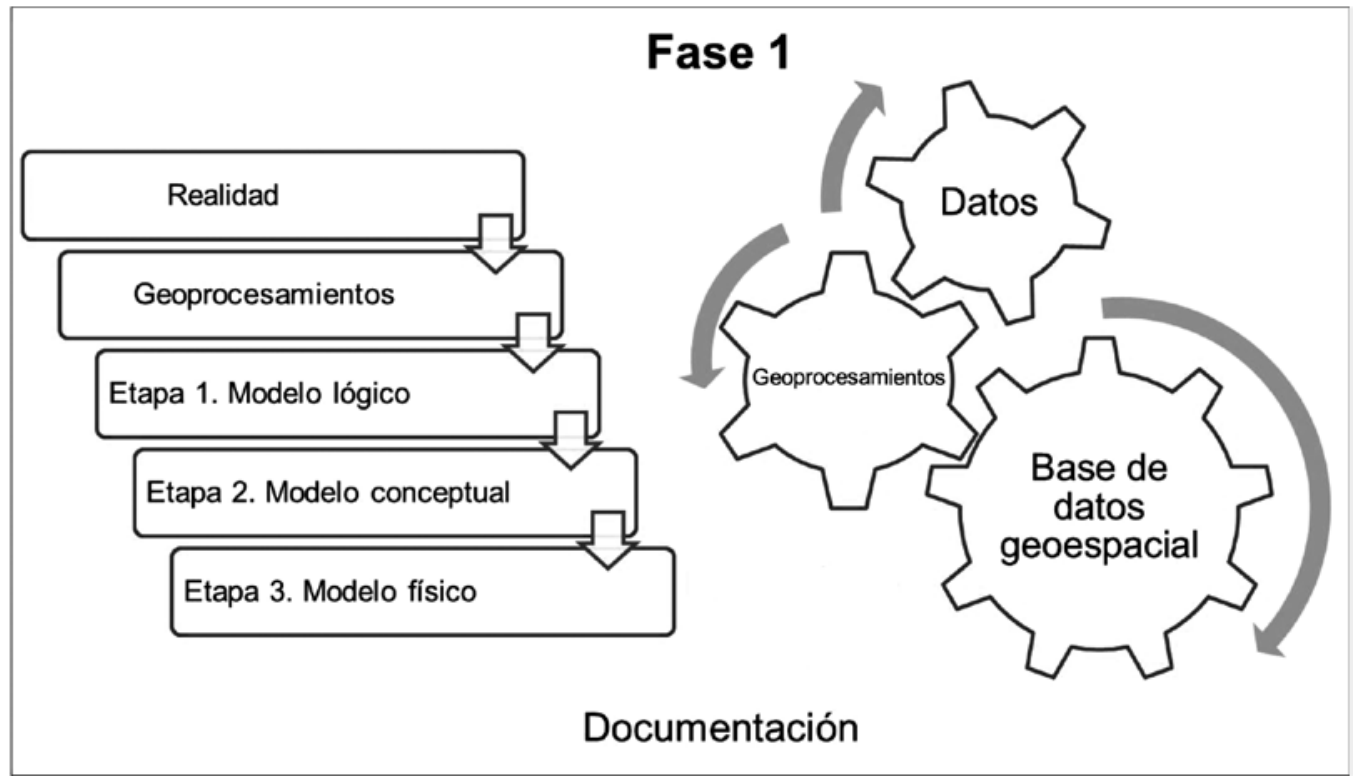

Fuente: Elaboración propia basado en el análisis metodológico.

La fase 1 es la planificación de diseño e implementación de la base de datos geoespacial, en el que se toman en cuenta los elementos necesarios para la gestión y estandarización del dato; los recursos humanos, tecnológicos y económicos, así como el estado actual del LHA. Para esto, es vital evaluar la realidad y visualizar los flujos de los geoprocesamientos que ilustren el escenario idóneo para la ejecución de la base de datos a partir del modelo conceptual, lógico y físico.

La fase 2 depende, directamente, de la estructura del repositorio de datos creado en la fase 1, debidamente documentado, en el que se dicta las pautas y lineamientos a seguir para presentar los productos en un visor de mapas en la web. 
Figura 3. Esquema general Fase 2

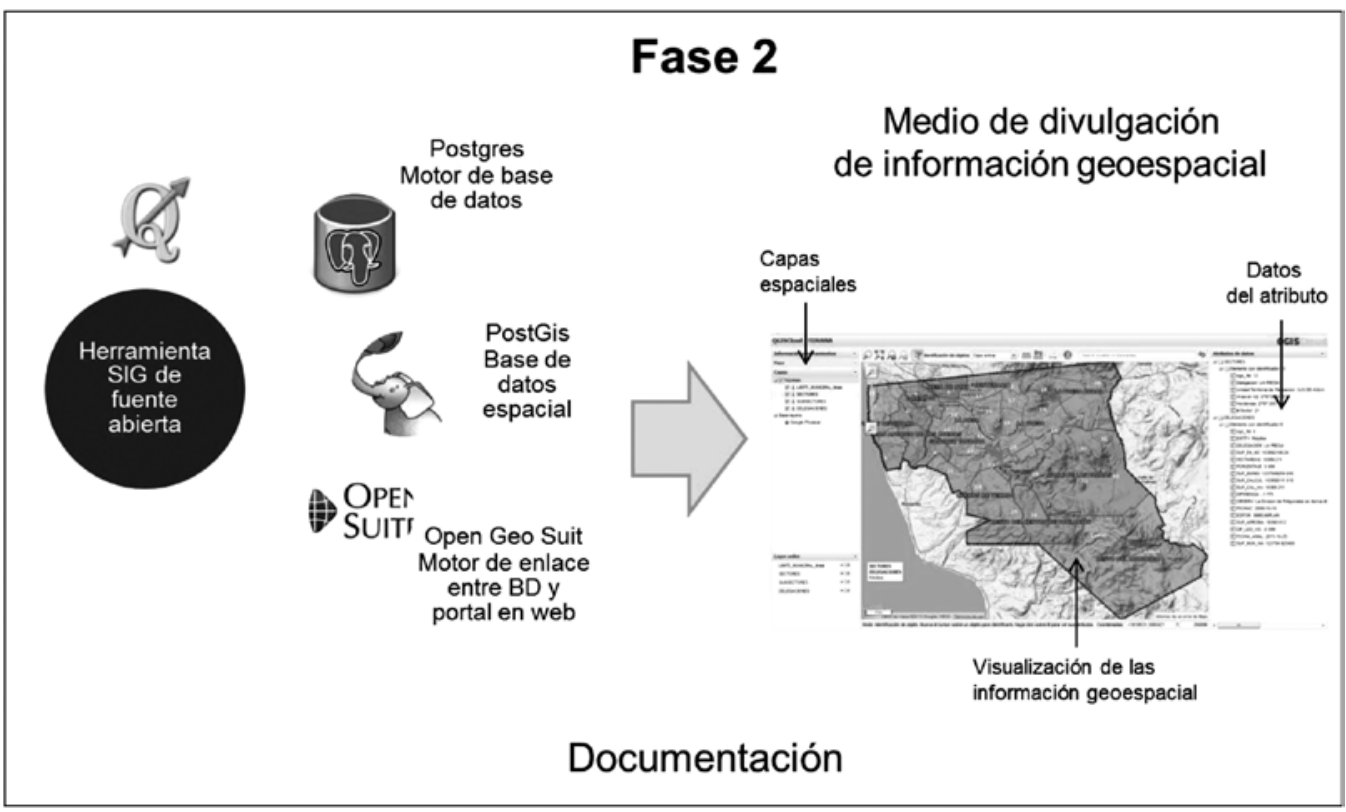

Fuente: Elaboración propia basado en el análisis metodológico.

La depuración, creación y relación de capas se generan en el programa QGIS, mismo que posee herramientas para el almacenamiento y gestión de información espacial manipulada por el motor de base de datos PostGress y PostGis.

Por su parte, el Geoportal para la divulgación estará ligado a la base de datos espaciales a través del sistema de servidor de mapas GeoServer y GeoExplorer, ambas herramientas agrupadas en la aplicación OpenGeo Suite, aplicación "diseñada y alojada de forma local, con la facilidad de colgar datos o proyectos de forma gratuita y sin mayores procedimientos de programación". (OpenGeo Suit, 2014, párr. 1, p.1).

Los protocolos de estandarización se apegan a la normativa ISOTC-211, este documento recoge los diversos temas concernientes a las reglas necesarias para el manejo y gestión de los datos geográficos. Esta actividad valida la depuración, orden, diseño, estructura, administración y catálogos de metadatos de la información geográfica, a través de las ISO 19100.

La información geográfica del proyecto, se extrae de los estudios realizados sobre el acuífero Jacó, específicamente de los siguientes aspectos: 
hidrogeológicos, uso del suelo, amenaza de contaminación de aguas subterráneas, vulnerabilidad acuífera, riesgo a la contaminación hidrogeológica, sitios de muestreos y resultados de laboratorio. Mientras tanto, los datos de cartografía básica como capas de ríos, curvas, calles y otros se extraen de los productos desarrollados por el Proyecto de Regularización de Catastro y Registro (PRCR), entre los años de 2005-2007. Se utiliza como herramienta al QGIS como la aplicación para la gestión de los datos geográficos.

El esquema de la figura 4, detalla el procedimiento secuencial del tratamiento del dato desde la fase de estandarización hasta lograr su publicación en una página web. La estructura es funcional como un sistema con las entradas de información, la retroalimentación o proceso en el repositorio de datos $\mathrm{y}$, por último, una salida con los productos para la toma de decisiones.

Figura 4. Proceso del dato dentro del sistema

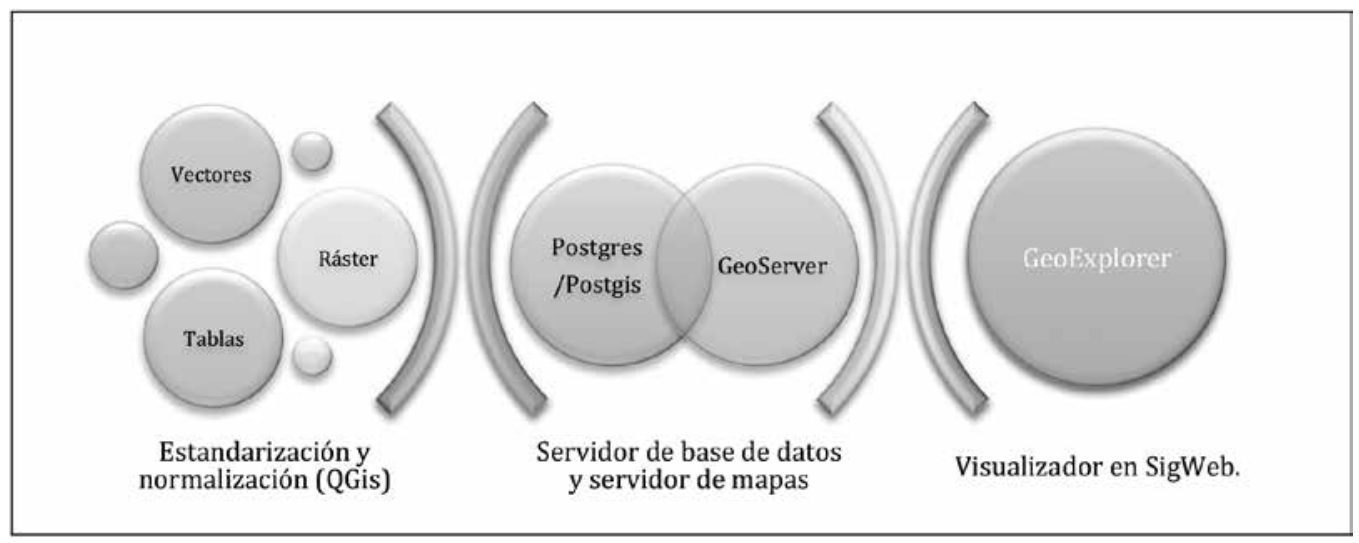

Fuente: Elaboración propia basado en el análisis metodológico.

Para los protocolos de estandarización de los insumos que alimentan al SIG y por ende, a la base de datos, se siguen una secuencia lógica previa a su almacenamiento, a esta sección se le conoce como el geoprocesamiento. Los datos pueden venir en formatos de tablas, mapas, cartografía digital o base de datos, se toma en cuenta que son productos desarrollados tanto por el laboratorio como por entidades externas.

De forma esquematizada, en la figura 5, con la ayuda de un flujo de procesos se detallan las etapas que deben cumplir los datos geográficos 
Christian Núñez Solis. Implementación de un visor geoespacial en la web sobre recurso hídrico para el acuífero Jacó, Garabito, Puntarenas, 2014

previos a la introducción a la base de datos geoespacial. Las actividades a realizar, son la depuración (eliminar errores), estandarización o/y normalización y visto bueno de la información.

Figura 5. Procedimiento de normalización de los datos cartográficos

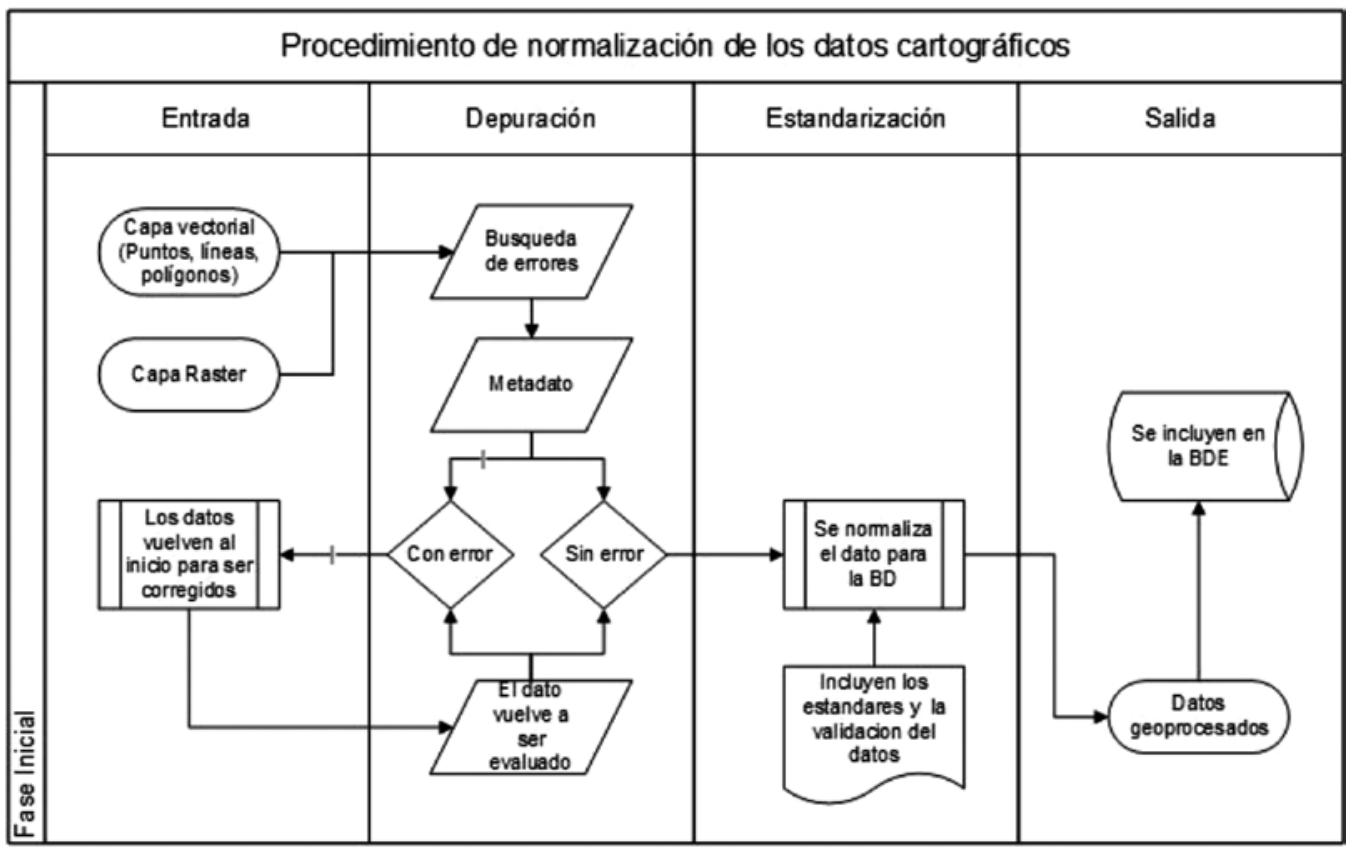

Fuente: Elaboración propia basado en el proceso de normalización.

Por su parte, como lo propone Elsmari y Navathe (2007), el diseño de la base de datos espacial seguirá los siguientes pasos para su creación: recopilación y análisis de requerimientos: se entrevista a los usuarios para entender y documentar sus requerimientos de datos. Además, se especifican los requerimientos funcionales que la aplicación debe implementar (operaciones o transacciones definidas por el usuario); Diseño conceptual: se crea el esquema conceptual usando un modelo de datos, para los propósitos de esta investigación el modelo entidad-relación sería el aplicado. Dicho esquema describe, detalladamente, tipos de entidades, relaciones entre ellas y restricciones. Paralelamente, se especifican las operaciones de usuario como transacciones de alto nivel; Diseño lógico (mapeo de modelo de datos): Se implementa la BD en PostgreSQL, a partir del mapeo 
del modelo entidad-relación y usando el modelo de datos específico del DBMS (modelo de datos de implementación), e.g., relacional u orientado a objetos, y Diseño físico: Se especifica la estructura de almacenamiento de los archivos, su organización, índices, y rutas de acceso. Simultáneamente, se diseñan e implementan los programas de aplicación correspondientes a las transacciones.

\section{Resultados}

Tres son los productos desarrollados al final de la investigación: la normalización del dato geográfico, la implementación de la base de datos y la implementación del Geoportal, cuyos resultados se explican a continuación:

Para la evaluación de calidad, normalización y validación del dato geográfico se utilizaron en total 24 capas geográficas como se enlistan en la tabla 1.

Tabla 1. Capas geográficas a utilizar en la investigación

\begin{tabular}{|l|l|l|l|l|l|}
\hline Número & \multicolumn{1}{|c|}{ Entidad } & \multicolumn{1}{|c|}{ Tipo } & Número & \multicolumn{1}{c|}{ Entidad } & \multicolumn{1}{|c|}{ Tipo } \\
\hline 1 & pozos & Puntos & 13 & distrito & Polígono \\
\hline 2 & colectoreslluvia & Puntos & 14 & geologia & Polígono \\
\hline 3 & aforos & Puntos & 15 & geomorfologia & Polígono \\
\hline 4 & pozoscr & Puntos & 16 & acuiferoscr & Polígono \\
\hline 5 & redvial & Línea & 17 & areaestudio & Polígono \\
\hline 6 & redhidrica & Línea & 18 & acuifero & Polígono \\
\hline 7 & curvasnivel & Línea & 19 & amenaza & Polígono \\
\hline 8 & equipotenciales & Línea & 20 & vulnerabilidad & \\
\hline 9 & lineasflujo & Línea & 21 & riesgo & Polígono \\
\hline 10 & rioacuiferorelacion & Línea & 22 & uso2007 & Polígono \\
\hline 11 & provincia & Polígono & 23 & uso2014 & Polígono \\
\hline 12 & canton & Polígono & 24 & ortofoto2007 & Ráster \\
\hline
\end{tabular}

Fuente: Elaboración propia a partir de los datos geográficos del laboratorio.

Para este producto se realizan las siguientes actividades: Comprobación del sistema de coordenadas: para los efectos del siguiente trabajo se utilizara la proyección CRTM05 con el datum WGS84; revisión en las propiedades del vector o el ráster, si existen un archivo de metadato 
asociado y completo, y una validación topológica del vector utilizando la herramienta "Comprobador de topologías" del programa QGIS. Los criterios a examinar son: a) en puntos: duplicados, b) en líneas: duplicadas, vértices sin conectar y sobre-puestas, y c) en polígonos: huecos, sobreposición y duplicados.

Una vez aplicada esta valoración a todas las capas en análisis se determina que: a) De las capas examinadas todas cumplen con la referencia geográfica, b) Del total de capas solamente los vectores de uso del suelo y ríos presentaron errores de sobreposición, duplicidad y vértices sin conectar, c) El dato geográfico tiene una consistencia en sus geometrías aceptable y puede ser introducida a la base de datos con confianza y d) Ningún archivo digital tiene asociado el metadato. Ante esta carencia es necesario desarrollar en CatMDEdit el registro pertinente siguiendo los requerimientos del ISO 19115.

Para las 22 capas geográficas en análisis se tuvo que realizar la edición de los metadatos, este repositorio queda a disposición para ser publicado a través de la web o para el uso del laboratorio. Con la certeza de contener un dato geográfico debidamente calificado, evaluado y con la consistencia necesaria, se procede a incorporarlo en la base de datos geográfica. 
Figura 6. Diseño conceptual de la base de datos geográfica

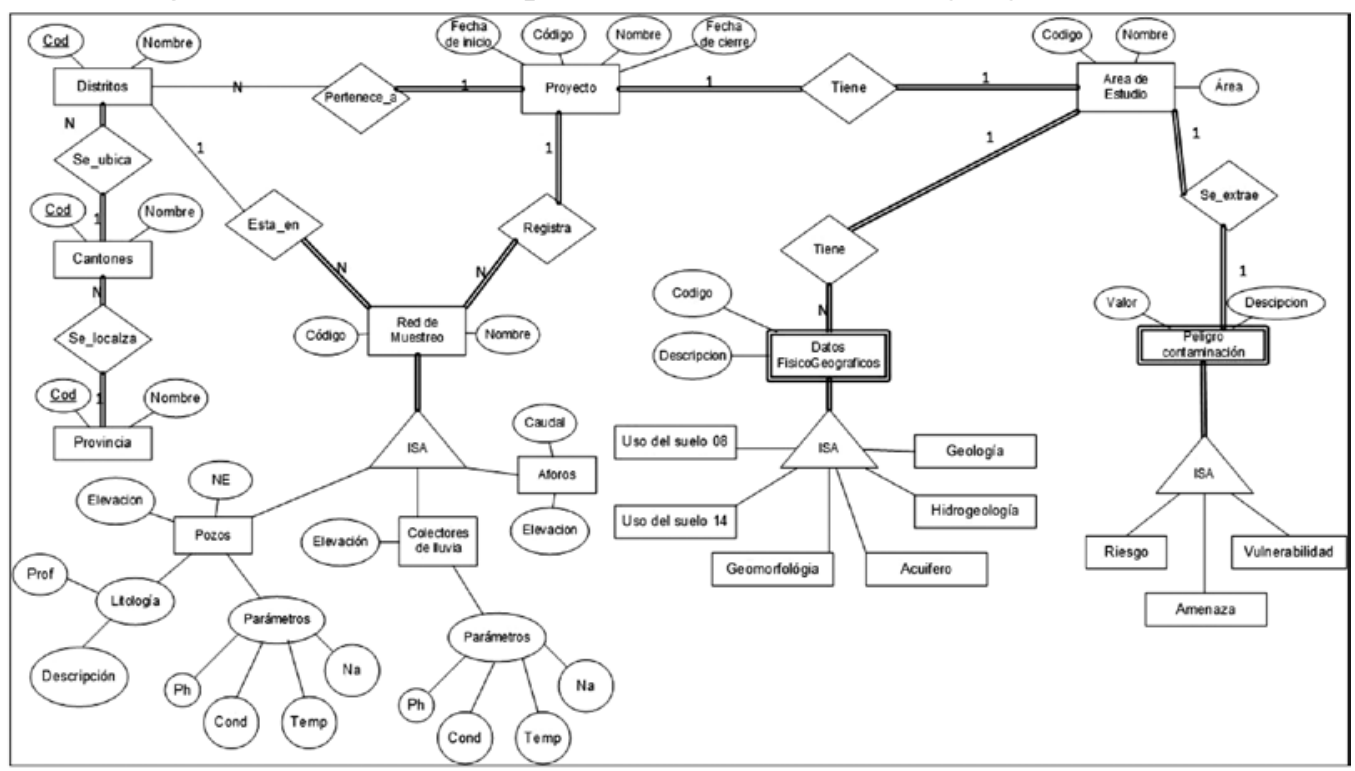

Fuente: Elaboración propia en el programa Visio.

El segundo producto, es la implementación de la base de datos. Como se ejemplifica en la figura 6, el diseño conceptual de la base de datos está encabezado por la entidad proyecto, de este elemento se relacionarán las entidades de localización (distrito, cantón, provincia), el área de estudio y la red de muestreo.

La red de muestreo, se especializa en un ISA que se compone de los pozos, colectores de lluvia y aforos, a los que se relacionan los parámetros de valoración de la calidad del agua.

Con respecto a la entidad del área de estudio, se relacionan dos entidades, los factores físico-geográficos y el peligro de contaminación.

Este modelo, es implementado en el motor de base de datos PostgreSQL, con la capacidad de gestionar los datos desde QGIS o divulgados en una conexión directa con OpenGeoSuit.

Una vez que es probado el repositorio de datos, se inicia la implementación del SIG Web. Dentro de esta etapa se desarrolla la conexión con la base de datos, la edición de los datos geográficos a publicar y la inserción dentro de un ambiente web geográfico para la visualización en 
internet. Se utiliza en esta actividad, las herramientas GeoServer y GeoExplorer, ambas contenidas en el paquete OpenGeoSuit. De forma resumida, la primera es un servidor de mapas para compartir, analizar y editar datos geoespaciales. Mientras que, la segunda es una aplicación para "la composición, el estilo, la edición y la publicación de mapas desde el navegador". (OPENGEOSUIT, 2014, párr.1, p.1).

Para la publicación de los datos en el Internet se utiliza GeoExplorer, que contiene las herramientas necesarias para enganchar los datos de forma directa a la web, sin procesos de programación o instructivos extensos de implementación. La secuencia para construir el módulo con las capas a visualizar (ver figura 7) se explican de la siguiente manera:

- La plataforma GeoExplorer debe conectarse al servidor de mapas GeoServer.

- $\quad$ En el punto 1, se añaden las capas que componen al mapa y en el punto 2, se conecta al GeoServer Local dando el visto bueno para la publicación.

- $\quad$ Si se quieren realizar modificaciones de estilo, etiquetas, colores con la herramienta de estilo dentro la misma página se realizan los cambios (punto 3).

- Como producto se obtiene un visor que incluye, además de las capas de la base de datos, coberturas externas de googlemaps y openstreet maps, herramienta de acercamiento, consulta, identificación, entre otras (punto 4). 
Figura 7. Creación del SIG Web

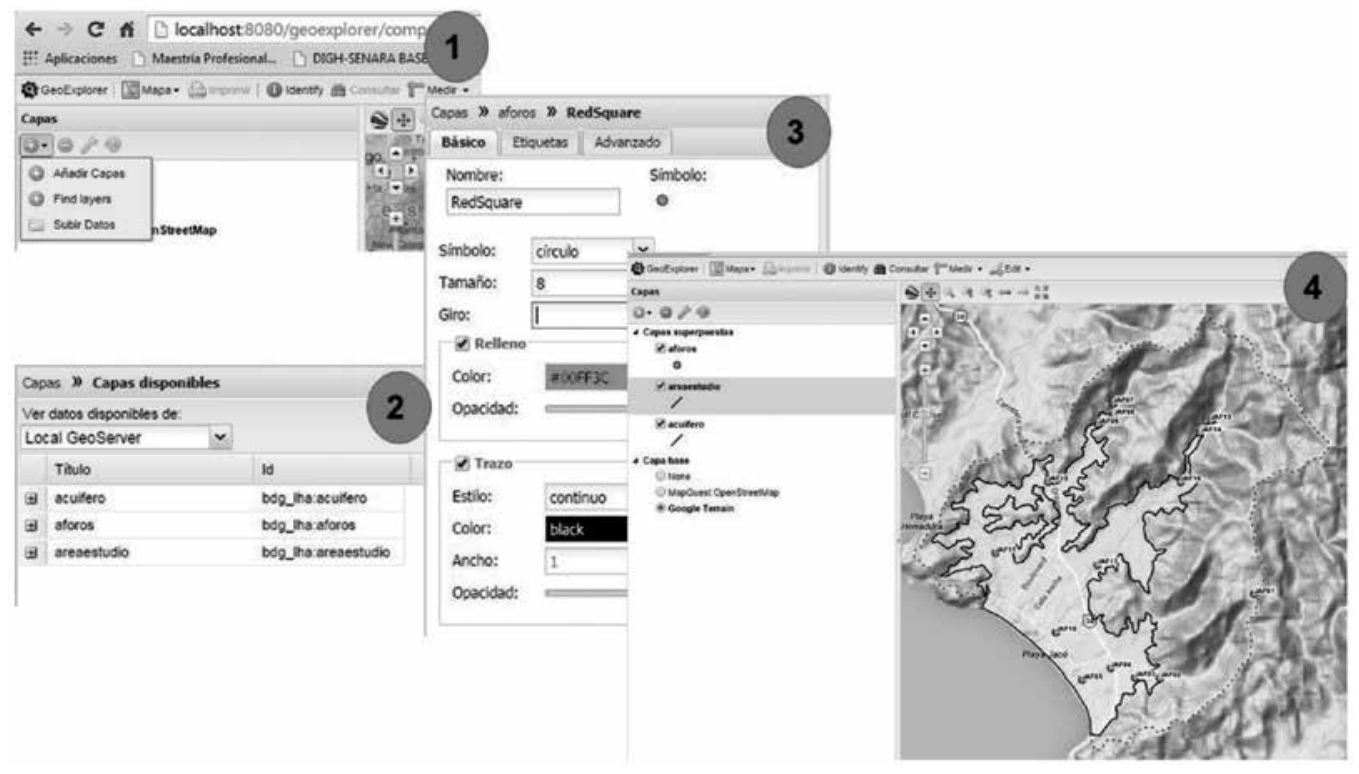

Una vez desarrolladas las capas a visualizar en el Geoportal, se construye un portal Web que sirve como plataforma para la consulta de los datos desarrollados en la investigación y que caracterizan al poblado de Jacó. La figura 8, ejemplifica la portada del SIG Web, el mismo se compone de un menú interactivo donde el usuario puede escoger entre mapas, publicaciones e imprimibles.

El sitio ha sido desglosado por temáticas según los datos geográficos: área de estudio, hidrogeología, físico-geográficos, red de muestreo, comparativo de uso del suelo y la evaluación del riesgo a la contaminación del recurso hídrico. El propósito es dar al usuario un espacio intuitivo y fácil de utilizar. 
Christian Núñez Solis. Implementación de un visor geoespacial en la web sobre recurso hídrico para el acuífero Jacó, Garabito, Puntarenas, 2014

Figura 8. Creación del SIG Web

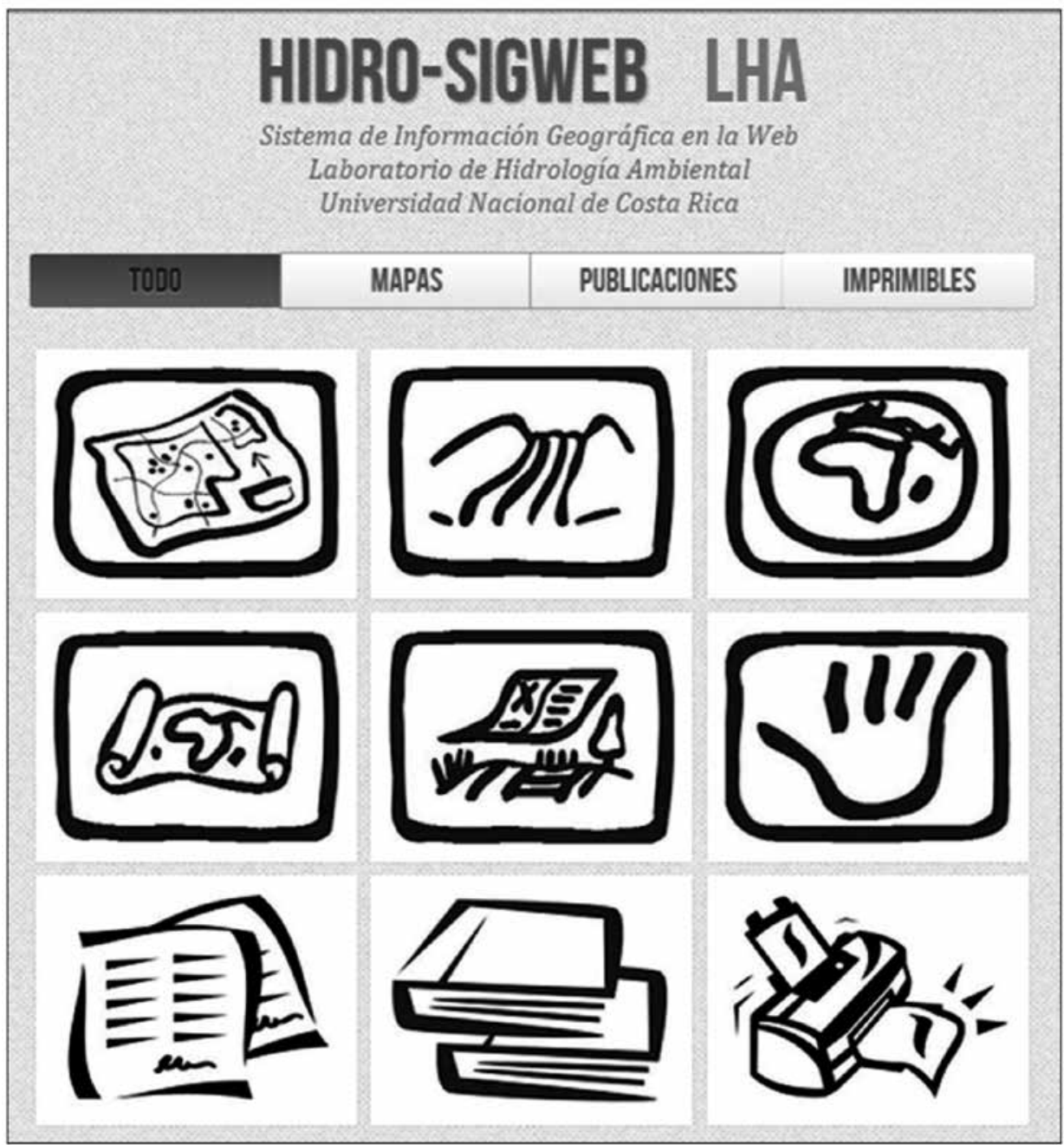

Cuando el usuario toca alguno de los botones se dirige al Geoportal con la temática seleccionada, en la figura 9 , se esquematiza un mosaico de imágenes con las capas geográficas inmersa en cada uno de los sitios del SIG Web. 
http://dx.doi.org/10.15359/rgac.1-54.2

Christian Núñez Solis. Implementation of geospatial web viewer regarding the water resource for the aquifer Jaco, Puntarenas, Garabito, 2014.

Figura 9. Creación del SIG Web

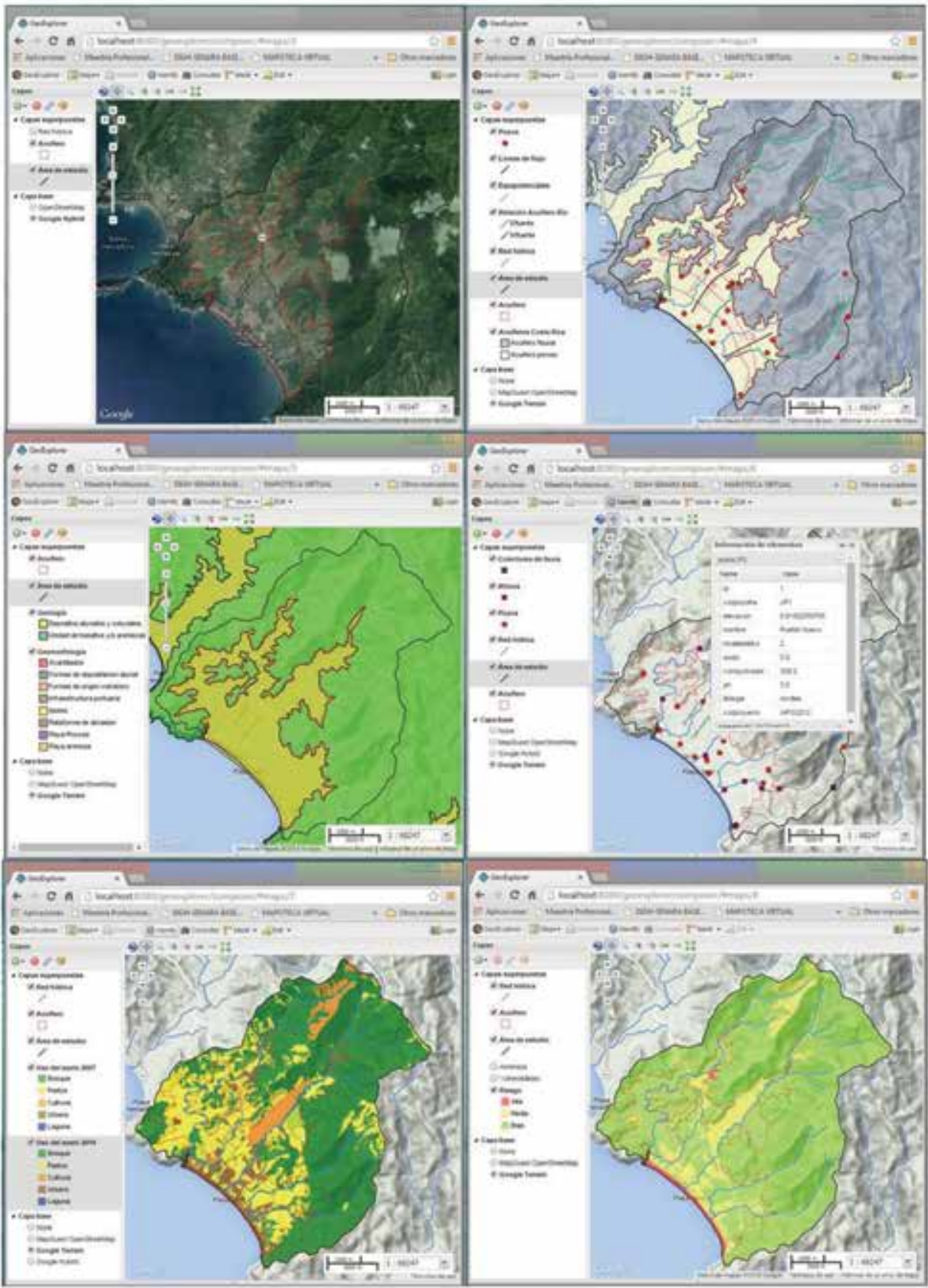




\section{Conclusiones}

La evaluación de la integridad, calidad y documentación del dato geográfico, es un eje primordial en las labores de cualquier ente desarrollador de información espacial. Sin embargo, el trabajo conlleva a una inversión considerable de recursos humanos, económicos y tiempo es por esto que debe incluirse en la planificación previa de un proyecto en SIG. Por ejemplo, para validar la cartografía de las 24 capas inmersas en esta área de estudio de $47 \mathrm{~km} 2$, le toma a una persona de 15 a 22 días a tiempo completo. Los trabajos que consumen mayor espacio son la corrección geométrica y la documentación del metadato.

El diseño e implementación de la base de datos debe ser evaluado por otros profesionales para así corregir inconsistencias, errores o disfuncionalidades. La retroalimentación y mejoramientos es un pilar en la planificación previa. Además, este repositorio de información debe estar construida para crecer y actualizarse, pues así lo requiere el laboratorio, los volúmenes de información geográficos son progresivos, cambiantes y nuevos.

El Geoportal, cumple con la función de informar y divulgar los estudios realizados por el laboratorio concerniente a la información geográfica. Sin embargo, es un prototipo que debe ser evaluado y mejorado, que no sea estático y si más dinámico, incluyendo nuevos temas, artículos o publicaciones; es decir, una página inteligente de comunicación para los interesados.

En general, se construye un producto de interés social y una herramienta facilitadora de procesos y toma de decisiones. Con la motivación de proveer a las comunidades investigadas un espacio de información y conocimiento de su territorio en el tema del recurso hídrico.

Los programas de código abierto utilizados, mostraron un alto potencial para cumplir con las tareas requeridas, son funcionales y confiables, esto los convierte en herramientas muy atractivas, no solo por su poco o nulo costo para implementarlos, sino por su estabilidad y fiabilidad.

Se recomienda como trabajo a futuro publicar los metadatos y la cartografía elaborada a través de otras plataforma de comunicación como los es geonetwork o dentro del Sistema Nacional de Información Territorial (SNIT) y que a su vez sean productos validados y de comunicación pública. 


\section{Referencias}

Anderson, G. y Moreno, R. (October 2003). Building Web-Based Spatial Information Solutions around Open Specifications and Open Source Software. Transactions in GIS, 7(4), 447- 466. doi: 10.1111/1467-9671.00158

Elsmari, R. y Navathe, S. (2007). Fundamentos de bases de datos. ( $\left.5^{\mathrm{a}} \mathrm{ed}.\right)$. Madrid, España: Pearson Educación SA.

Gorni, D., Giannotti, M., Brito, P., Knopik, A. y Rodríguez, M. (2007). Sistema de información geográfica para web con informaciones espaciales (geográficas) de output e input para expediciones. A I Jornadas de SIG Libre. Girona: Universitat. Recuperado de http://dugi-doc.udg.edu/bitstream/handle/10256/1233/SistInfoWeb_Com. pdf? sequence $=1$

Instituto Geográfico Nacional. (1983-1989). Hojas cartográficas Herradura y Tárcoles. Escala 1:50 000. Proyección Cónica Conforme de Lambet. San José: Instituto Geográfico Nacional, Costa Rica.

Instituto Panamericano de Geografía e Historia (IPGH). (2010). Guía de normas. Comité ISO/TC-211. Información geográfica/geomática. Edición en español. México, DF.: Instituto Panamericano de Geografía e Historia.

Instituto Tecnológico de Costa Rica. (2008). Atlas digital de Costa Rica. Escala 1:200 000. Proyección Transversal de Mercator CRTM05. Cartago: Instituto Tecnológico de Costa Rica.

Olaya, V. (2011). Sistemas de información geográfica. España: OSGEO. Recuperado de http://wiki.osgeo.org/wiki/Libro_SIG

OpenGeoSuit. (2014). Información general. Recuperado de http://boundlessgeo.com/solutions/opengeo-suite/. Fecha de consulta: 27 de abril de 2014

OpenSourceOrg. (2014). Open Source Iniciative. Recuperado de http:// www.opensource.org/

Radilla, F. (2008). Modelado de datos para base de datos espaciales. Caso de estudio: sistemas de información geográfica. Maestro en Ciencias en la Especialidad de Ingeniería Eléctrica. México, DF: Centro de Investigación y de Estudios Avanzados del Instituto Politécnico Nacional, Departamento de Computación. 
Sánchez, Y. y Amoros, M. (2012). Gestión del agua urbana mediante Análisis Espacial en los SIG. Ingeniería Hidráulica y Ambiental (RIHA), 33(3), 58-71.

Tomlinson, R. (2007). Pensando en el SIG. New York, Estados Unidos: ESRI.

Unidad Ejecutora del Proyecto Regularización Catastral y de Registro. (2007). Cartografia base: línea de costa, curvas de nivel, ríos y carreteras. Escala 1:5000. Proyección Transversal de Mercator CRTM05. San José: Asamblea Legislativa, Costa Rica.

Unidad Ejecutora del Proyecto Regularización Catastral y de Registro. (2007). Ortofotos del área de Jacó. Escala 1:5000. Proyección Transversal de Mercator CRTM05. San José: Asamblea Legislativa, Costa Rica. 